\title{
How Does Eco-Coaching Help to Save Energy? Assessing a Recommendation System for Energy-Efficient Thermostat Scheduling
}

\author{
Rayoung Yang ${ }^{1}$, Devika Pisharoty ${ }^{2}$, Soodeh Montazeri ${ }^{3}$, Kamin Whitehouse ${ }^{2}$, Mark W. Newman ${ }^{1}$ \\ ${ }^{1}$ School of Information, University of Michigan, MI, USA \{rayang, mwnewman\}@umich.edu \\ ${ }^{2}$ Computer Science Department, University of Virginia, VA, USA \{dnp6ga, whitehouse\}@virginia.edu \\ ${ }^{3}$ Fors Marsh Group, VA, USA smontazeri@forsmarshgroup.com
}

\begin{abstract}
This paper presents findings from a field deployment that explored a design approach we call eco-coaching: giving personalized suggestions for specific actions that would reduce wasted energy. We studied ThermoCoach, which performs eco-coaching for thermostat scheduling. It senses and models occupancy patterns in a home, and provides occupants alternative suggestions for configuring their thermostat. Our study shows that eco-coaching accomplished four things. First, it made it easier for users to implement an effective thermostat schedule. Second, it supported user agency in negotiating energy savings and comfort goals. Third, it facilitated learning different scheduling strategies as well as weighing different options. Finally, it challenged users' beliefs about how well they were doing. These outcomes, in turn, were successful in getting users to employ and experiment with more efficient setback strategies. Going forward, we propose ways that eco-coaching systems could better support users in customizing and assessing the systems' recommendations.
\end{abstract}

\section{Author Keywords}

Sustainability; Thermostat; Eco-Coaching; Energy Savings

\section{ACM Classification Keywords}

H.5.m. Information interfaces and presentation (e.g., HCI): Miscellaneous.

\section{INTRODUCTION}

Many believe that technology can play a key role in helping people consume less energy, but there are competing approaches to achieving this goal. Eco-feedback techniques inform people about their own energy usage in order to empower and motivate them to make better decisions and consume less energy [6]. For example, some systems tell

Permission to make digital or hard copies of all or part of this work for personal or classroom use is granted without fee provided that copies are not made or distributed for profit or commercial advantage and that copies bear this notice and the full citation on the first page. Copyrights for components of this work owned by others than the author(s) must be honored. Abstracting with credit is permitted. To copy otherwise, or republish, to post on servers or to redistribute to lists, requires prior specific permission and/or a fee. Request permissions from Permissions@acm.org.

UbiComp '16, September 12-16, 2016, Heidelberg, Germany Copyright is held by the owner/author(s). Publication rights licensed to ACM. ACM 978-1-4503-4461-6/16/09...\$15.00

DOI: http://dx.doi.org/10.1145/2971648.2971698 people how much they are consuming [13] and in some cases present a breakdown of how they are consuming it [25]. However, information alone is not always enough. Some energy saving actions are too complex or time consuming for people to do them regularly. Therefore, automation techniques seek to take action to save energy on behalf of the user. This approach has been explored extensively for things like lighting control [21], thermostat control [19], and even vehicular route navigation [8]. While automated systems have shown promise in limited field trials, recent studies found evidence that showed that autonomous systems did not work as successfully as expected in the real world [31, 32].

In this paper, we examine a different approach that we call "eco-coaching": giving personalized suggestions for specific actions that would reduce wasted energy. Ecocoaching systems assist users with energy savings, but leave users in control. We propose that eco-coaching should go one step farther than eco-feedback: it should not only provide feedback on energy consumption in the past, but also leverage user behavior in order to identify waste and recommend actions to prevent energy waste in the future. However, eco-coaching should stop one step short of automation: it should identify actions that can reduce waste but should not take them on behalf of the user. Using a mixed-initiative approach [10], eco-coaching extends both eco-feedback and automation to balance system autonomy and user control in thermostat scheduling [16,19,24,32].

ThermoCoach [24] was designed to provide eco-coaching for thermostat control. It first monitors users' behavior and energy use patterns over time and identifies areas for improvement to reduce energy waste. It then generates and emails personalized and actionable schedule recommendations to users and makes it easier for them to take action. To assist users in balancing their energy savings and comfort goals, it presents eco-feedforward messages to provide information about projected savings and comfort expectations for each recommendation. Finally, ThermoCoach allows users to customize the recommended schedules it provides. This is especially useful in cases where the system could not identify particular needs or situations, such as preferences for sleep temperature or the presence of pets. 
To evaluate the impacts on energy savings, a 12-week field deployment was conducted, comparing the energy saving outcomes of ThermoCoach with two other approaches: manual programming and automatic scheduling. As previously reported, results of a ThermoCoach field deployment study indicated that eco-coaching saved $4.7 \%$ more energy than manual programming and $12.4 \%$ more energy than automation [24]. The present paper is based on a new analysis of interview data collected as part of the previously published field deployment study. In particular, we focus the experience of eco-coaching from the user's perspective, and examine how ThermoCoach's ecocoaching features influenced users to save energy.

We found that the eco-coaching approach 1) made it easier for users to implement an effective thermostat schedule, 2) supported user agency in negotiating trade-offs between energy savings and comfort, 3) facilitated learning different scheduling strategies, and 4) challenged users' beliefs about how well they were doing. These outcomes, in turn, were successful in getting users to employ and experiment with more efficient setback strategies. While our initial results are promising, we also find room for improvement, especially in supporting users to assess recommendations. In particular, evaluating the fit and performance of the recommendations is important for building user trust, thereby increasing acceptance and maximizing benefits of eco-coaching recommendations.

\section{RELATED WORK}

We summarize key approaches in designing systems to promote energy conservation. First, eco-feedback aims to help users to be aware of their energy use and make informed decisions to save energy. Second, automation tries to reduce the workload for users by automating tasks that users need to do manually. Third, mixed initiative approaches seek to balance system capability and human control to address shortcomings of previous approaches.

Eco-feedback displays data to inform users of their consumption of various resources, such as electricity, gas or water, and thus seeks to motivate users to change their behavior. While eco-feedback has been shown to increase awareness of resource consumption, several studies that investigated everyday practices of consumption $[15,23,29,32]$ found that obtaining information did not actually trigger people to take action or change behavior. Strengers warned against a common assumption that the eco-feedback approach holds, depicting the user as "a resource man" who makes rational choices and acts accordingly when provided information [30].

Automation approaches seek to relieve user burdens by automating users' tasks. For example, research on occupancy-based thermostat control (e.g., $[9,26]$ ) seeks to detect and/or predict when the home is unoccupied so that the thermostat can be set to an optimally efficient level, and return to the occupants' preferred comfort level before they are likely to return. The Nest thermostat takes a different approach by learning users' adjustments and automatically generating a schedule instead of modeling the occupancy pattern [33]. With the advancement and availability of wearable and smart home sensing devices, Huang et al. [11] investigated the possibility of developing new models of thermal comfort to generate a real-time predictive comfort model [5] instead of or in addition to the occupancy pattern or temperature adjustment pattern.

While these systems have shown promise in limited field trials, there remains a need to understand how such "smart" features will interact with users' desire for control and predictability. Recently, several studies investigated users' lived experience of smart systems and observed evidence that autonomous systems did not work as successfully as expected in the real world [27,31]. These studies showed that systems often fell short of anticipating or responding to dynamically changing everyday life situations. Some users became frustrated and even abandoned the smart devices when they did not understand how those devices worked or why they did not function as expected [2,18,20,31]. In response to smart system shortcomings such as lack of understanding of nuanced and dynamically changing situations in the real world and users' loss of control, several projects have proposed mixed-initiative approaches [10] to balance system autonomy and user control in thermostat scheduling $[16,19,24,32]$. While the notion that systems could recommend energy saving actions has been proposed before $[7,12,14]$, the approach has not previously been studied through deployments in the home environment.

More recently, several research projects have designed and evaluated agent-based systems that provide suggestions to conserve energy by utilizing dynamic pricing and renewable energy $[1,4,28]$. These systems process a large amount of data such as weather forecasts, peak loads on the power grid, and renewable energy generation to figure out the best time to perform energy-consuming activities. Then, these systems prompt users to use appliances such as laundry machines or dishwashers at times when the electricity pricing is cheaper or when they can use renewable energy. Costanza, et al. [4] conducted a simulation study in which a washing machine agent allowed users to book a time to do their laundry to save cost. They found that some participants who had structured routines for doing laundry found it easier to fit the system into their existing practice. However, other participants found it challenging to plan for their laundry as they usually ran their washing machine as needed. Bourgeois, et al. [1] employed a series of interventions such as energy feedback messages, proactive suggestions and direct user control to support users with photovoltaic solar energy generation to plan their laundry on daily basis. They found that participants perceived proactive suggestions to be more useful than feedback messages, although they did not necessarily follow the suggestions. Simm, et al. [28] designed a system to forecast renewable energy generation 
for a local community. They found that participants were able to make use of the information and that some participants actively shifted times when they did laundry or dishwashing to maximize their use of green energy.

In this paper, we study ThermoCoach (previously published in [24]), which provides multiple personalized and actionable suggestions for thermostat scheduling. It differs from the aforementioned systems $[1,4,28]$ in that it provides personalized recommendations based on individual homes' characteristics instead of making general suggestions based on power grid load or green energy generation.

\section{ECO-COACHING DESIGN APPROACH}

Here, we describe the eco-coaching approach that guided the design of ThermoCoach [24]. We define eco-coaching as giving personalized suggestions for specific actions that would reduce wasted energy while leaving users in control of those actions. Below are specific eco-coaching features of ThermoCoach. Figure 1 shows the ThermoCoach email, in which following eco-coaching features are incorporated.

Personalized recommendations: The system monitors occupancy patterns of a home over time using Bluetoothbased occupancy sensing and IR-based motion sensing. It builds a model indicating the probability that the home is occupied at each time of the day. Then, it generates schedule options that fit the occupancy pattern of the house and reduce energy waste. The recommended schedules maintain the typical temperature settings preferred by each household, but generates setback strategies by adding new setback temperatures for times when the house tends to be unoccupied. Three scheduling choices differ in the amount of time the system remains at the setback temperature level and therefore represent different tradeoffs between predicted comfort and energy savings. Details about the recommendation algorithm can be found in [19] and [24].

Eco-feedforward: To assist users in making informed decisions, the system provides eco-feedforward messages to convey the projected impacts of the recommended changes [32] in terms of energy savings (i.e., 5\%, 7\% and 10\%) and expected comfort level (i.e., Barely change, May decrease slightly, and Will decrease). This supports users in exercising their agency to negotiate priorities between energy savings and comfort preferences.

User control: Instead of letting the system automatically change the schedule, the system leaves users in control by asking them to review schedule options and make decisions about which recommendation to follow (if any) and how schedule options should be implemented. Users can adjust schedule options to complement what is not considered by the system and better accommodate their preferences.

Easy invocation: Oftentimes users do not have the time or willingness to program their thermostats. The system sends a 'push' email containing different recommendations. Users can instantly initiate a new schedule by clicking a button in the email.

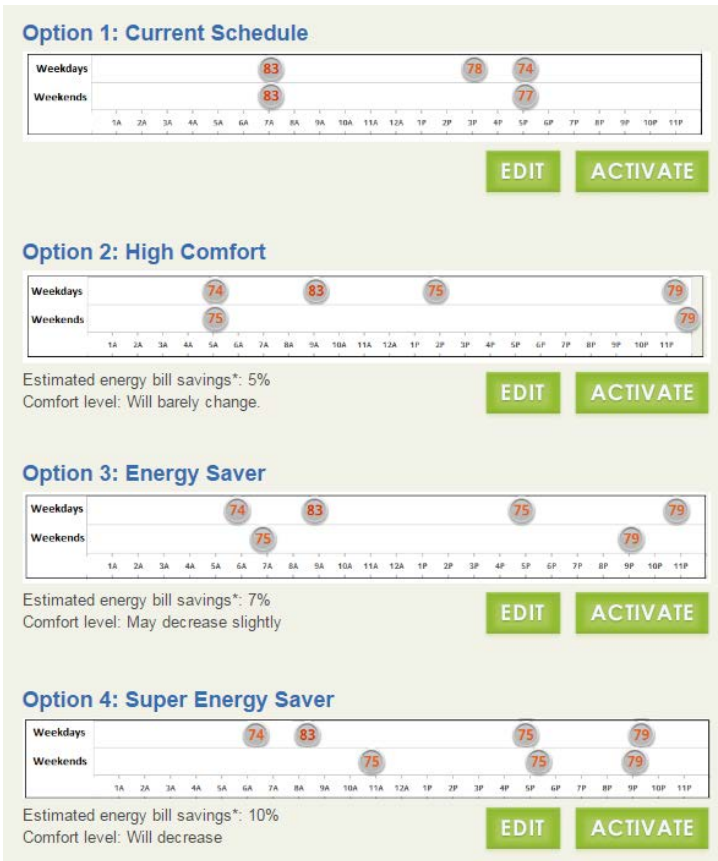

Figure 1. ThermoCoach emails four options to each user: their current schedule, a high comfort schedule, an energy saver schedule, and a super energy saver schedule. Users can Activate a schedule by clicking a button to have it automatically programmed into their thermostat. If users click the Edit button, they are brought to a web page (Figure 2 below) that allows them to edit schedule options.

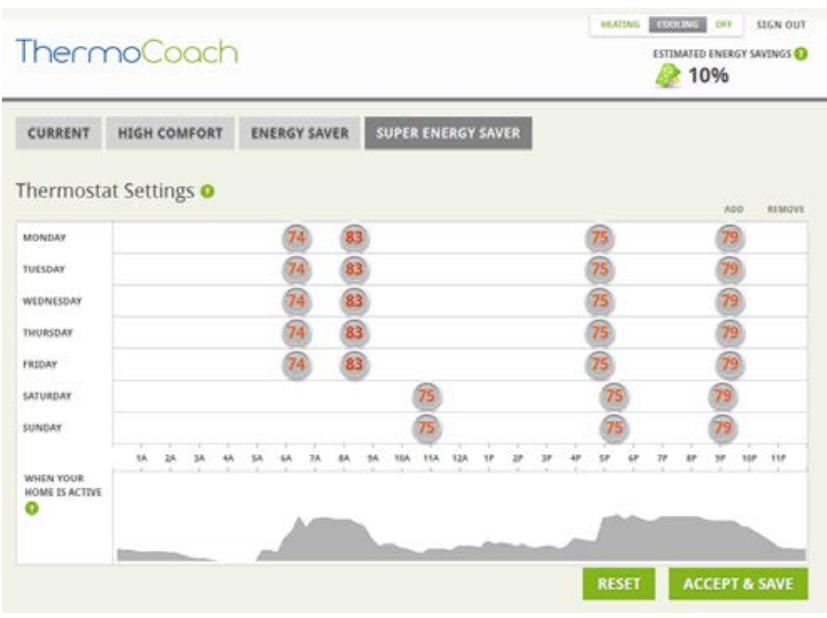

Figure 2. ThermoCoach web page allows users to view and edit detailed thermostat schedule options. Users can select a schedule option from menu (top left). While editing, users are informed of estimated energy savings (top right) based on the current settings. The graph along the bottom shows the times when their home is typically active.

\section{METHODS}

A previous paper reported on an analysis of the sensor data, thermostat usage, and ThermoCoach interaction, showing that ThermoCoach had an impact on energy savings [24]. 
That paper, however, did not investigate why ThermoCoach impacted users' choices. For that, we turned to the interview data to study how different types of mechanism affect participants' energy saving and thermostat scheduling practices.

\section{Participants}

Over 27,000 flyers and door hangers were distributed through local newspapers and by manually placement on doorknobs to recruit participants. After screening and dropouts, 36 households participated in the interviews associated with this study. All homes were located within 30 miles of each other and were subject to similar weather conditions throughout the study. Table 1 below describes characteristics of those households.

Table 1. Key characteristics of the 36 households

\begin{tabular}{|c|c|}
\hline $\begin{array}{l}\text { Housing } \\
\text { type }\end{array}$ & $\begin{array}{l}32 \text { homes own a single family house, } 4 \text { own/rent an } \\
\text { apartment, condo or town house. }\end{array}$ \\
\hline Education & $\begin{array}{l}25 \text { homes with Master's or Ph.D., } 11 \text { homes with } \\
\text { B.A/B.S or Associate's degree. }\end{array}$ \\
\hline $\begin{array}{l}\text { Number } \\
\text { of } \\
\text { occupants }\end{array}$ & $\begin{array}{l}21 \text { homes have adults with children under } 18 \text { years old. } \\
13 \text { homes have only adults. } 2 \text { homes are single person } \\
\text { homes. }\end{array}$ \\
\hline Pets & 25 homes have pets (e.g., dogs, cats, fish, rabbits). \\
\hline $\begin{array}{l}\text { Average } \\
\text { summer } \\
\text { energy bill }\end{array}$ & $\begin{array}{l}24 \text { homes: ranged between } \$ 100 \sim \$ 200 ; \\
4 \text { homes: above } \$ 200 ; 4 \text { homes: under } \$ 100 ; \\
4 \text { homes did not respond. }\end{array}$ \\
\hline
\end{tabular}

\section{FIELD DEPLOYMENT STUDY}

At the end of May 2014, we started instrumenting participants' homes with data collection endpoints, motion sensors, Bluetooth transmitter tags, and Nest thermostats. By the end of June, all instrumentation was completed. Participants were divided into three groups to compare the energy saving impacts among three thermostat scheduling approaches: manual programming, automation, and ecocoaching. As shown in Table 2, each group used a different thermostat scheduling approach. Group P and Group TC manually programmed their Nest thermostat. Group N used their Nest thermostat with built-in automation features that automatically programmed the schedule ("Auto-Schedule") and adjusted the temperature when motion was not detected for a certain amount of time ("Auto-Away").

Table 2. The participant homes were divided into three groups. All groups received eco-feedback emails. Group $P$ could only manually program their thermostat. Group $\mathbf{N}$ used Nest's automation features. Group TC received eco-coaching recommendations (Figure 1).

\begin{tabular}{|l|l|l|l|}
\hline & Group P & Group N & Group TC \\
\hline $\begin{array}{l}\text { Eco-feedback } \\
\text { (Nest monthly Energy Report) }\end{array}$ & $\sqrt{ }$ & $\sqrt{ }$ & $\sqrt{ }$ \\
\hline Auto-Schedule, Auto-Away & - & $\sqrt{ }$ & - \\
\hline Recommendations & - & - & $\sqrt{ }$ \\
\hline
\end{tabular}

In August 2014 we performed an intervention event. We sent an email to all households with Nest's monthly Energy Report. Additionally, households in Group TC were emailed ThermoCoach schedule recommendations and were asked to respond to the recommendations within 48 hours. The Nest Energy Report included eco-feedback elements such as the number of hours the air conditioner ran, the number of days that the Nest detected the home was unoccupied and activated an energy-saving mode called "Auto-Away," and the number of days a home earned a green leaf by adjusting the temperature to save energy. All homes received their second monthly Nest Energy Report in September. More details on participant recruitment and field deployment study methods are reported in [18].

\section{INTERVIEW STUDY}

As a part of the field study, a series of interviews were conducted. Each participant home participated in three interviews during the study period. The first interviews were conducted before we installed the Nest thermostat at each participant' home. During the first interview, we asked participants about their existing thermostat control practices, their satisfaction or issues with their current thermostat, and their attitudes towards energy savings. The second interview was conducted after participants used their newly installed Nest thermostat more than a month. We asked participants in all groups questions about how they experienced the Nest thermostat focusing on whether and how participants' thermostat control practices changed after using the Nest's eco-feedback features, graphical user interface, and remote control. For Group N, we additionally asked about their experience of the Auto-Schedule and Auto-Away features. After the second interviews, we performed the intervention. The final interview was conducted at the end of the study, roughly two months after the intervention. During the final interviews, we asked participants about how their scheduling practices changed during the study and their response to the Nest Energy Report. With Group TC participants, we probed their reactions to the ThermoCoach recommendations, how they responded to the recommendations and why, and how ThermoCoach changed their thermostat control practices.

Each interview took approximately $30 \sim 45$ minutes. When it was possible, we interviewed all adult occupants in each household. All interviews were conducted by phone, audiorecorded, and transcribed. We analyzed 108 interviews from 36 homes that completed their participation in the interview study. The interview transcripts were coded and analyzed using an iterative process of generating, refining, and probing the themes that emerged. Codes were initially drawn from research questions and then supplemented with those that emerged from the interviews. A key interest in this study was to observe participants' scheduling practices with different control mechanisms: manual programming, automation, and eco-coaching. We focused on understanding how ThermoCoach recommendations influenced participants' thermostat scheduling and control practices changes in Group TC (eco-coaching), as compared to participants in Group P (manual programming) and Group N (automation) who did not receive schedule recommendations. 


\section{FINDINGS}

Before we report findings from the interview study, we briefly summarize the findings from the quantitative comparison of the three approaches, which were previously published in [24].

- Group TC saved more energy than the other two groups. By receiving eco-coaching recommendations, this group was able to save $4.7 \%$ more energy than Group $\mathrm{P}$, which used the Nest without automation features, and 12.4\% more energy than Group N, which used the Nest automation ("Auto-Schedule").

- $\quad$ Eight out of 13 homes in Group TC adopted a new schedule based on the received recommendations. Six of these eight homes had a schedule without setbacks prior to the intervention and activated a schedule with new setbacks afterwards. Two homes already had a schedule with setbacks, but activated a more energy efficient schedule.

These results indicated that Group TC homes adopted new and more efficient setback temperatures than homes in the other groups and thus saved more energy. In the following sections, we draw on interview data to describe Group TC participants' experiences and reflections on ThermoCoach's eco-coaching features. We refer to participants by pseudonym. Unless indicated otherwise, participants were from Group TC.

\section{Making it easy to take action}

Push recommendations via email made it easy to implement suggestions and concrete schedule options reduced the time and effort required to generate a schedule.

Actionable recommendations allowed users to take action. Participants liked that the recommendations provided them actionable and concrete plans for future savings. Most participants reported that the recommendations allowed them to recognize opportunities to save energy and indicated what to do, making it easier to take action. Many, like Amy, considered prospective, proactive plans useful: "The Nest monthly report reflects on the past month, which is nice. ...The [ThermoCoach] recommendations are nice because they're thinking in the future, and they're looking at your patterns and saying, 'Well, based on what you've got it set at now, these options would be totally doable.",

Personalized recommendations increased credibility and reduced uncertainty.

Generating an effective schedule required reconstructing the daily ins and outs and the nuances of users' comfort needs. When participants saw that the recommendations reflected their occupancy patterns and followed their temperature settings, they liked the recommendations since they were tailored to their specific home. Tom described ThermoCoach as somebody who works for him: "I loved it because somebody was thinking for me. Absolutely. ...We could have done it, but ...it was just really nice for somebody else to evaluate how we used the house, and then to make suggestions." When participants recognized that ThermoCoach recommendations were tailored to their particular household's situations, they considered the recommendations credible and relevant. They felt ThermoCoach was not asking them to follow a random schedule as it reflected their existing patterns and temperature preferences. This made participants more willing to adopt a recommended option.

\section{Supporting user agency}

A core design principle of eco-coaching is leaving users in charge of making decisions about how to balance their energy savings and comfort needs while providing assistance to guide their decision making. Group TC participants described ways in which ThermoCoach supported user agency in their thermostat scheduling.

Having a choice provided sense of control in negotiating energy savings and comfort goals.

Three schedule recommendations, in addition to users' current schedule, allowed participants to review and compare different options and make decisions about whether to opt-in and which option to choose. Several participants reported that having a choice between different schedule options gave them a sense of control. Jessica explained that she felt she was in charge: "You guys weren't just telling me, 'Here's the best way for you to proceed. Do this!' But really, putting the ownership on us felt like we were taking charge of it and taking charge of our own actions. So I liked that." Multiple recommendations (High Comfort, Energy Saver and Super Energy Saver) allowed participants to negotiate their energy saving and comfort goals according to their motivations and priorities. Interestingly, changes in life situations affected participants' motivations and priorities, and offering multiple recommendations allowed them to negotiate their goals according to the change. For example, Amy chose a 'High Comfort' option for her husband since he had to stay home for the summer due to an injury.

Eco-feedforward supported decision-making by presenting quantified estimates of future savings.

ThermoCoach provides eco-feedforward, which presents quantified energy savings estimates $(5 \%, 7 \%$ and $10 \%)$ coupled with comfort levels (Barely change, May decrease slightly and Will decrease). Finding a sweet spot between energy savings and comfort is a key consideration in setting a thermostat schedule. Therefore, participants found this combination useful when deciding which option to choose. In particular, quantified measures made energy savings more tangible and practical when justifying their decisions. Jim explained how eco-feedforward information helped him make decisions: "It does give you an indication, and it certainly helps to quantify. ...It's a little bit harder to actually quantify the energy savings looking at the bill because you've got the other variable of your weather changes as well. But just basing it off of sheerly comfort and knowing that ...you should anticipate this type of savings, I think that's a very effective combination." 
Admittedly, participants acknowledged that predicted energy savings were difficult to estimate due to many changing variables such as daily weather, physical conditions, and daily routines. Liz assumed that the estimation of energy savings was calculated based on the average temperature of her location. She further explained that while eco-feedforward information did not guarantee the energy reduction that was estimated, it was useful for deciding which schedule to choose: "As a consumer, as long as I knew that it was just an estimate and there was no guarantee that I was gonna save X amount of dollars, then I think that might have some weight or bearing on what option that I choose."

As suggested in [32], we observed in this study that ecofeedforward prompted participants' motivation for setting a new goal. For example, Mike was influenced to change his thermostat schedule to save more energy. He chose the 'High Comfort' option. He found it was comfortable and stayed with a more energy efficient schedule: "What [ThermoCoach recommendations] did tell me is that ...had I made those slight changes, I should still be fairly comfortable and I should also notice some savings. And again, the difficulty is, because all things aren't equal, 'Did in fact that happen?' Well it's hard to say. But, at the same time, I haven't gone back and modified the settings that were set up with that option ['High Comfort']."

\section{Customization allowed ways to accommodate preferences.}

ThermoCoach creates schedule options to maximize setbacks and provides schedule options that increased setbacks. While prompting users to adopt new, more efficient setbacks, we found that ThermoCoach also supported user control by allowing users to accommodate their particular preferences and needs through editing the schedule. This was found to be effective in increasing the adoption of recommendations. For example, four homes modified schedule options to better fit to their individual home and still made their schedule more efficient than before. One had a fish tank and changed the setback temperature for the daytime. Two homes chose a 'High Comfort' option, which made minimal changes to their schedule, but delayed the cooling start time a little bit. As a result, the changed schedule was still more efficient than their previous schedule.

\section{Encouraging experiments with a setback strategy}

As mentioned earlier, the previously reported results [24] indicated that more participants in Group TC employed higher (i.e., more efficient) setback temperatures than other groups. As part of the present analysis, we performed additional data analysis and found a notable difference between Group TC and other groups. There were 15 homes that initially had a schedule that kept one single temperature all times, among the 36 homes across all groups. Interestingly, six such homes in Group TC that received recommendations all adopted new setbacks. On the other hand, none of the other nine homes changed their schedules. They were in Groups $\mathrm{P}$ and $\mathrm{N}$ and did not receive ThermoCoach recommendations. In this section, we explain how ThermoCoach recommendations were successful in encouraging participants to experiment with the schedule and adopt new higher setback temperatures.

Comparison of schedule options facilitated learning of different scheduling strategies and weighing pros and cons.

Placing participants' existing schedules alongside different schedule recommendations initiated quick reflection and provided participants with learning opportunities. Many participants described recognizing similarities and differences between schedules easily as they looked through different schedule options. For example, they noticed how time and temperature settings varied among different options. This helped some participants to gain insights into different ways to employ setback temperatures. For example, Amy had a night setting that she set to cool at $75^{\circ} \mathrm{F}$ throughout the night. When she found that recommended schedule options suggested raising the setting to $79^{\circ} \mathrm{F}$ in the middle of the night, she reflected on her existing schedule and contemplated an idea to create a new setback that she had not considered previously: "We probably wouldn't be able to feel a difference during that sleeping pattern to feel the four-degree difference. So, you could save energy without affecting comfort, essentially. That was good to know."

Offering a more comfortable option lowered the barrier to acceptance.

Providing the 'High Comfort' option along with more aggressive plans helped to lower barriers to adopting a new setback that was higher than the existing schedule. Because the 'High Comfort' option did not make dramatic changes from participants' existing schedule, having this option eased participants' concerns or uncertainty during the process of adopting a new schedule. For example, some participants were not comfortable going for the more aggressive option at first. However, they were willing to try the "High Comfort" option since it was not "that big of a hard shift," as Jessica described. Also, participants who started with the 'High Comfort' shared that such an option could help them gradually transition to a more aggressive option.

Alternative options challenged users' beliefs and triggered users to experiment with a new schedule.

When participants were presented with new schedule recommendations, existing beliefs that might have hindered them from increasing the energy efficiency of their schedule were often challenged. One of the notable benefits of recommendations is supporting participants in correcting their misconceptions and encouraging them to experiment with a new schedule. For example, Jim as noted earlier opted for 'High Comfort,' had grown up being taught, "Leave the thermostat at one setting. That's the most efficient thing to do." He kept the schedule mostly at $76^{\circ} \mathrm{F}$ as shown in Figure 3. When Jim received ThermoCoach recommendations, he found that the idea of a setback 
increasing energy savings was contrary to what he had always been taught. However, he decided to see how it would work and chose the 'High Comfort' option, which was the most conservative approach amongst the three options. His changed schedule is shown in Figure 4.

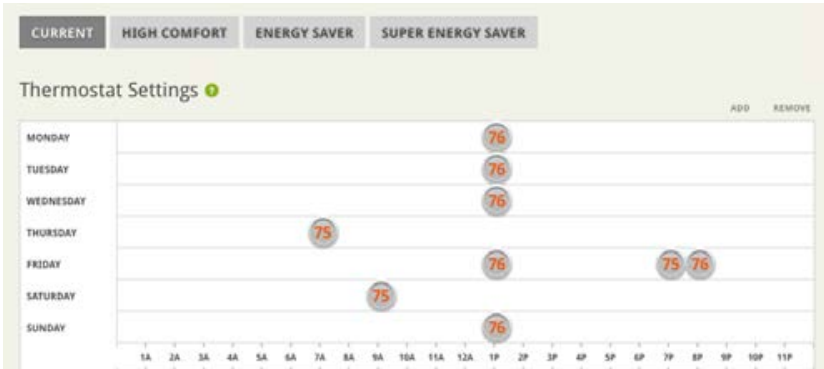

Figure 3. Jim's previous schedule kept the temperature mostly at $76^{\circ} \mathrm{F}$ all times.

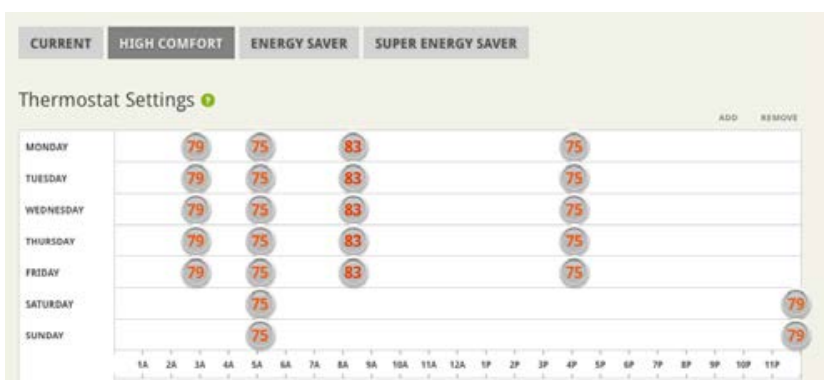

Figure 4. Jim adopted the 'High Comfort' option, which included two new setbacks; one was $8^{\circ} \mathrm{F}$ degrees higher and the other was $4^{\circ} \mathrm{F}$ higher than his normal temperature.

Jim explained that recommendations helped him overcome a certain reluctance to adjust the settings of his system:

"This was a very interesting way to be able to experience that change or experience a result of altering those settings without fear of putting in a completely inappropriate setting, if you will. I thought that was a very beneficial way of doing it, whereas if I was going to try and just do this on my own, I may not have been as well versed in terms of knowing what it would do." Jim also added that, "I would be more likely to experiment with that again to see if I could boost my savings and keeping my comfort level comparable to where it is. "Like Jim, Tom found that recommendations were helpful because "otherwise, you would just leave it at what you had because that was comfortable, not realizing that a slight change can result in a real saving without a real impact to your comfort level."

On the other hand, Steven kept the temperature at $75^{\circ} \mathrm{F}$ all day. When he looked the projected energy savings that the recommendations proposed, he was still uncertain about the idea of adding a setback. Therefore, he accepted the 'Super Energy Saver' option, but adjusted the setback temperatures to stay between $76^{\circ} \mathrm{F}$ and $78^{\circ} \mathrm{F}$. He explained why he changed the setback temperatures: "Just kind of my limited knowledge. It seems like that takes more energy than just trying to keep a house at a steady state, but maybe I'm wrong about that."

\section{Shortcomings of ThermoCoach}

As we reported, ThermoCoach recommendations provided various benefits and assisted participants with improving the energy efficiency of their thermostat schedule. In this section, we report shortcomings and limitations of ThermoCoach recommendations based on participants' insights and reflections.

Inability to assess the performance of recommendations lowered user trust in system and its recommendations.

Participants expressed their desire to check how effective the recommendation they chose was in delivering energy savings. For example, Patrick implemented the 'Super Energy Saver' option to save $10 \%$, the greatest savings among all schedule options. However, Patrick found that it was not straightforward to know whether he indeed achieved the $10 \%$ energy savings that he anticipated. He explained that he could not trust the recommendations if he was not able to verify the actual energy savings after using the schedule: "Because you can recommend, you can tell me I'm gonna get $10 \%$ savings if I choose option D. Or, a $1 \%$ savings if I choose option $B$. Or, no savings if I continue with option A. You can tell me that. But, I'm not gonna believe you until you actually give me some statistics that says, 'For the entire month of June, you used 100 kilowatt hours. In the month July, you only used 87 kilowatt hours. Which represented actually a 13\% savings, and we estimated it would be about a 10\%.' I need to be able to hear something as concrete as that before I have any confidence that I'm actually going to achieve something just because you tell me that I will."

As mentioned earlier, participants acknowledged that it is difficult to estimate energy savings when there are various factors that are dynamically changing. Thus, they did not expect the actual outcomes to match the estimates exactly. However, participants expected the system to provide concrete evidence to allow them to assess the effectiveness of implementing the recommendations. Without such evidence, they might not have trust or confidence that they would achieve the desired savings by following the system's recommendations.

Inability to detect a mismatch between the schedule and actual use missed opportunities for savings.

In addition to assessing the performance of the recommendation after it was implemented, it would also have been useful to assess how the recommendation was working while it was active. Liz accepted a recommended schedule and thought the schedule worked well for her home. She did not change the schedule after activation. However, during the final interview, she found that the schedule she activated had a setback temperature of $83^{\circ} \mathrm{F}$, which was higher than she expected. After learning about this, Liz remembered the times when she noticed an $83^{\circ} \mathrm{F}$ setting on the Nest thermostat and she simply kept turning it down. It did not occur to her that she might need to check the schedule. Liz suggested that ThermoCoach should provide a new recommendation if users kept making 
overrides without realizing that their schedule was not working for them: "I think some follow-up email would probably be nice to tell me that you're not really abiding by this recommendation. ...Then if the new email could perhaps say that, 'You are consistently overriding the recommendations,' and maybe suggest some new recommendations with maybe lower temperatures or an adjustment of the schedule somehow that still saves energy, but makes me more comfortable, like optimize the process. I think that would be helpful. I would probably respond really well to that also."

Failing to address user preferences decreased acceptance of recommendations.

Five out of 13 homes did not opt-in to any option and four homes edited recommendations before they activated them. Here, we explain reasons that those participants did not adopt schedules ThermoCoach recommended or edited schedule options before activated them. First, the setback temperatures recommended were too high for some. ThermoCoach suggested daytime setbacks that were 4 or 8 degrees higher than the regular temperature of each household. Some homes were already making efforts to save energy by having higher temperatures, such as $80^{\circ} \mathrm{F}$, as setbacks or even as their regular temperature. Therefore, those homes thought an even greater setback temperature such as $88^{\circ} \mathrm{F}$ was too extreme. Second, many households had pets, but ThermoCoach did not take pets into consideration and when creating setbacks for times when there was no human occupancy. For example, Laura had rabbits and kept the temperature at $79^{\circ} \mathrm{F}$ during the day. When recommendations suggested a setback temperature of $87^{\circ} \mathrm{F}$, she found that they were "so drastically" high. Third, participants prioritized comfort needs. Some homes mentioned that it was difficult for them to sleep if it was not cool when going to bed. Emma "vetoed" the recommendations since they suggested raising the nighttime temperature by four degrees. She explained that it would be uncomfortable for her family: "It was the one that said that we should raise our temperature at night, and we said, 'No way.' ...I'm already having hot flashes." Finally, two participants said that they were simply too busy. One commented that it would be rare for her to find a half an hour to set a new thermostat schedule. Another did not remember receiving the ThermoCoach email.

Two homes experienced discomfort after they accepted a recommendation. Nora found that her fish tank was looking unhappy. Her husband found that the setback temperature was too high for the fish tank and adjusted the schedule. In Patrick's home, his wife felt it was quite warm when she returned home. Patrick lowered the setback temperature. Both homes fixed the problem by revising their schedule. One home may have had a system or networking error. This home chose a Super Energy Saver schedule, but the activation did not work due to an unknown error. They did not realize that they were using their old schedule until the final interview.
In summary, we have just described the benefits and shortcomings of ThermoCoach recommendations as they aimed to assist participants in improving their thermostat scheduling while supporting participants' agency. We found that ThermoCoach supported participants in employing new higher setbacks to increase energy savings and in managing the tension between energy savings and comfort goals. Next, we discuss the effectiveness and shortcomings of the design features of ThermoCoach. Then we propose design implications for eco-coaching systems to better assist users with planning, executing and assessing their thermostat scheduling effectively.

\section{DISCUSSION}

ThermoCoach follows the core principle of eco-coaching: giving personalized suggestions for specific actions that would reduce wasted energy. ThermoCoach employs several concrete design features to perform eco-coaching for thermostat scheduling. Our findings show that this ecocoaching approach accomplished four things. First, it made it easier for users to implement an effective thermostat schedule. Second, it supported user agency in negotiating competing energy savings and comfort goals. Third, it facilitated learning different scheduling strategies as well as weighing pros and cons of different options. Finally, it challenged users' beliefs about how well they were doing. In the following sections, we highlight challenges involved in designing recommendation-based eco-coaching systems like ThermoCoach.

\section{Creating recommendations: Improving personalization}

In personalizing schedule options for each household, ThermoCoach focused on two key aspects that greatly varied in individual households - occupancy pattern and comfort preferences. To generate a model for occupancy patterns, ThermoCoach collected data using various sensors for six weeks. Participants particularly liked that ThermoCoach collected data over time and because of this they considered the schedule options to be credible. Inferring comfort preferences was important because people might ignore a recommendation if it simply asked them to raise the temperature setting to save energy without considering their comfort. Thus, the system used existing temperature settings in users' schedules as a way to incorporate their comfort needs. These design choices were found to be effective in generating recommendations that fit the occupancy patterns and accommodated the comfort needs of individual households.

In designing ThermoCoach, we decided to leave users in control of revising schedule options to meet any additional situations and comfort needs, such as maintaining cooler air for pets and at nighttime. However, it could be more effective to consider certain variables for personalization to better understand their constraints and requirements for a thermostat schedule. We suggest that a system like ThermoCoach should ask users for information regarding, for example, pets and sleep preferences. 
Having pets was a common factor to consider for scheduling. About $70 \%$ of participants in this study had pets. According to the 2015-2016 APPA National Pet Owners Survey, $65 \%$ of U.S. households own a pet [34]. Therefore, considering pets when generating schedule recommendations would be useful. For households with younger children, comfort needs were also prioritized over energy savings. Interestingly, some households commented that they would have opted-in to more aggressive options for the winter schedule because the energy bill tended to be much higher in winter than summer for participants in our study. One participant, who was in Group P, lost her job during the study and wanted to use less cooling to save money. Generating options to address changes in energy saving motivations should be considered to increase the benefit of eco-coaching. For the successful adoption of recommendation-based eco-coaching systems like ThermoCoach, it is critical to allow users to complement the system's inability to understand or anticipate varied and changing needs and situations in individual homes. Doing so can also improve the performance of the system and increase satisfaction with the recommendations.

\section{Assessing recommendations: Multi-phase assessment} The ThermoCoach system provides estimated energy savings as a way to prompt users to adopt a schedule recommendation. However, it does not report how much following the recommendations actually saved. One participant mentioned that he would not trust the system unless it provided concrete evidence to show if or to what extent the recommendations worked as the system had proposed. To build user trust with recommendation-based eco-coaching systems, systems should support users to assess the quality and performance of recommendations over time. We suggest that an eco-coaching system should provide not only projected energy savings estimation for the future, but also quick and easy assessment of how effective the recommendations were at delivering what they proposed to users after implementation.

Assessing the actual performance of recommendations after use with consideration of real-world factors and conditions

While assessments would be useful, there is a challenge in evaluating the performance of recommendations-the actual energy saving outcomes - compared to the estimated savings. There are various factors that dynamically change in real environments that cannot be predicted in advance. For example, weather changes throughout the season and people's daily schedule and activities vary; these in return affect how people heat/cool their house, and physiological and psychological factors affect their comfort preferences. Indeed, many participants in our study reported that such variability was a major reason they were unable to assess their energy efficiency since they could not simply compare their energy bills month-by-month or year-by-year.

Here, we note that several of our participants also mentioned that they understood that estimated savings were 'estimated,' and that actual savings would vary according to changing conditions and situations in the real environment, such as weather. What these participants wanted was information that helped them believe the system would bring the benefits it proposed as long as the conditions under which the estimates were made were maintained. Thus, it is important for an eco-coaching system to indicate to what extent the actual energy savings out- or underperforms the initial estimation. Then, the system should also explain what factors affect the differences between actual and estimated savings.

Providing information regarding to what extent and in what aspects the actual conditions and user behaviors in the real environment were different from the predicted conditions and user behavior pattern would be useful. The most obvious factor would be weather. The system could show how the weather differed from previous months. It could also show how occupancy patterns were different than the patterns that were used to generate the initial recommendations. This way, the system could better support users to reflect on how the difference impacted the heating or cooling needs of the house. As we mentioned earlier, one household had one member who had to stay home due to injury, greatly increasing the amount of time during which that house was occupied.

Providing hindsight evidence with post-hoc simulation of alternative recommendations

We suggested that assessment of the performance of the recommendations after use would be beneficial. However, there would still be a lack of evidence that the recommendation was particularly good because there would be no way to compare the recommendations to an alternative. Users do not know what might have been if they had implemented other schedule options. It would be useful for users to be able to evaluate not only the recommendations they chose, but also alternatives that they did not implement.

We propose that eco-coaching systems should compare how alternatives might have worked compared to the recommendation they used. Eco-coaching systems could provide post-hoc simulations for the alterative options along with assessment of the chosen option to gauge the outcomes that might have been achieved. Understandably, users do not have all the necessary information to know which option would work mostly effectively for them at one time. However, it becomes much easier to gauge how different strategies would have worked afterwards.

Providing post-hoc assessment of the alternatives could be effective in providing opportunities to understand how different strategies would have worked. In particular, it would make it easier for users to correct their existing misconceptions and thus make more informed decisions for future scheduling. For example, Steven, who did not choose a schedule option with a higher setback temperature, might have been convinced if he had seen how alternative options could have worked under the same circumstances. 
Assessment of alternatives using post-hoc simulation would provide additional evidence to reinforce the performance of a chosen recommendation because it is easier to evaluate performances that are comparable to each other. More importantly, this would provide an additional learning opportunity for users to discover pros and cons of different options in accommodating various everyday situations.

Hindsight is always 20/20. While projected estimation was useful for users in making decisions for their planning, reflecting on how their schedule worked as well as how alternatives might have worked could inform users about the impact their decisions had or might have had. We note that users would have reacted differently to alternatives if they were indeed used; for example, users might have felt more uncomfortable and ended up overriding the temperature and consuming more energy. Simulations of alternatives would be still useful for users in reflecting on their decisions and possible outcomes.

\section{Performing assessment for the schedule in use}

So far, we have discussed the benefit of assessing recommendations after implementation. Lastly, we suggest that an eco-coaching system should conduct an ongoing assessment of the performance of the schedule that is in use. One of the functions that we described for an ecocoaching system includes monitoring user behaviors and their energy use and identifying discrepancies between them. In addition to monitoring user behavior and energy use to generate recommendations, an eco-coaching system should perform quick, ongoing checks to assess how the schedule in use is working.

For example, as in the case of Liz, if users are making many overrides after accepting a recommendation, an ecocoaching system can follow up and ask if the users want to stay with their choice or if the system should provide new recommendations based on their adjustments to the schedule. This would provide an opportunity to discover time or temperature settings that do not work for users. If users happen to be not committed to their decisions (e.g., a schedule they chose based on their motivations for energy savings), follow-up checks could be an opportunity for them to choose a more realistic option. The system would also need to monitor how the schedule is working in terms of occupancy patterns. When users' occupancy patterns change from those upon which the recommendations were based, the system should generate new recommendations to adjust to the modified needs for the home.

If provided, tools enabling users to assess the quality and performance of recommendations, including the schedule they used as well as alternatives, would increase the credibility of recommendations over time. This, in turn, would allow users to trust the system and further experiment with their scheduling to increase energy savings. In addition, this process could support users in exercising their discretion (knowledge and insights into different situations that were not sensed or interpreted by the system) to better evaluate the quality and performance of different strategies based on their particular situations. Users would be able to build and strengthen their understanding and ability to utilize and apply various strategies for scheduling to accommodate their interests and priorities.

\section{LIMITATIONS}

Our studies were restricted to a certain geographic region (Virginia, United States), and looked at a restricted set of people over a constrained period of time. We were only able to send one recommendation during one summer season due to challenges with the deployment. It will be valuable for future research to explore the long-term interaction with eco-coaching systems like ThermoCoach. Our participants were highly educated, likely to have their own house, and were mostly married. Most of them were motivated to save energy. Less motivated users might be less inclined than our participants to respond to ThermoCoach schedule recommendations. The 36 homes in our study were not representative of the vast diversity of living situations found in US residences - to say nothing of differences across the globe. More research is needed to consider the difference in individual homes and to validate if this approach could work for a larger group of people.

ThermoCoach takes a somewhat conservative approach to achieving a balance between energy efficiency and comfort in the sense that it treats "comfort" as a relatively stable state that can be mapped consistently onto a specific temperature. Other recent work $[3,17]$ has challenged this approach to comfort, for example by applying models of Adaptive Thermal Comfort [22]. We believe adaptive models are compatible with an eco-coaching approach (for example, adaptive behaviors could be included as recommendations within ThermoCoach) and feel that this is a promising direction for future research.

\section{CONCLUSIONS}

Eco-coaching assists users by providing recommendations tailored to their behavior patterns and preferences and making it easier to take actions, but also leaves users to make decisions about whether or how they should follow such recommendations. Our study participants identified several benefits of ThermoCoach. It made it easier for them to generate a schedule, provided opportunities to reflect on their thermostat schedule by comparing alternative options and weighing pros and cons, and helped them to make informed decisions for individual homes' needs and situations. Further, it challenged their existing beliefs and encouraged experimenting with their scheduling.

\section{ACKNOWLEDGMENTS}

Rayoung Yang was supported by a Rackham Predoctoral Fellowship from the Rackham Graduate School at the University of Michigan. This work was funded in part by NSF grant IIS-1149601 and NSF grant 1305362. 


\section{REFERENCES}

1. Jacky Bourgeois, Janet van der Linden, Gerd Kortuem, Blaine A. Price, and Christopher Rimmer. 2014. Conversations with my washing machine: an in-thewild study of demand shifting with self-generated energy. In Proceedings of the 2014 ACM International Joint Conference on Pervasive and Ubiquitous Computing (UbiComp '14). ACM, New York, NY, USA, 459-470.

DOI=http://dx.doi.org/10.1145/2632048.2632106

2. A.J. Bernheim Brush, Bongshin Lee, Ratul Mahajan, Sharad Agarwal, Stefan Saroiu, and Colin Dixon. 2011. Home automation in the wild: challenges and opportunities. In Proceedings of the SIGCHI Conference on Human Factors in Computing Systems (CHI '11). ACM, New York, NY, USA, 2115-2124. DOI=http://dx.doi.org/10.1145/1978942.1979249

3. Adrian Clear, Adrian Friday, Mike Hazas, and Carolynne Lord. 2014. Catch my drift?: achieving comfort more sustainably in conventionally heated buildings. In Proceedings of the 2014 conference on Designing interactive systems (DIS '14). ACM, New York, NY, USA, 1015-1024. DOI: http://dx.doi.org/10.1145/2598510.2598529

4. Enrico Costanza, Joel E. Fischer, James A. Colley, Tom Rodden, Sarvapali D. Ramchurn, and Nicholas R. Jennings. 2014. Doing the laundry with agents: a field trial of a future smart energy system in the home. In Proceedings of the SIGCHI Conference on Human Factors in Computing Systems (CHI '14). ACM, New York, NY, USA, 813-822. DOI=http://dx.doi.org/10.1145/2556288.2557167

5. Mark Feldmeier and Joseph Paradiso. 2010. Personalized HVAC control system. Internet of Things (IOT), 2010, IEEE, 1-8.

6. Jon Froehlich, Leah Findlater, and James Landay. 2010. The design of eco-feedback technology. In Proceedings of the SIGCHI Conference on Human Factors in Computing Systems (CHI '10). ACM, New York, NY, USA, 1999-2008.

DOI=http://dx.doi.org/10.1145/1753326.1753629

7. Silvia Gabrielli, Paula Forbes, Antti Jylhä, et al. 2014. Design challenges in motivating change for sustainable urban mobility. Computers in Human Behavior 41, 416-423. DOI $=$ http://dx.doi.org/10.1016/j.chb.2014.05.026

8. Raghu K. Ganti, Nam Pham, Hossein Ahmadi, Saurabh Nangia, and Tarek F. Abdelzaher. 2010. GreenGPS: a participatory sensing fuel-efficient maps application. In Proceedings of the 8th international conference on Mobile systems, applications, and services (MobiSys '10). ACM, New York, NY, USA, 151-164. DOI=http://dx.doi.org/10.1145/1814433.1814450

9. Manu Gupta, Stephen S. Intille, and Kent Larson. 2009. Adding GPS-Control to Traditional Thermostats: An Exploration of Potential Energy Savings and Design Challenges. In Proceedings of the 7th
International Conference on Pervasive Computing (Pervasive '09), Hideyuki Tokuda, Michael Beigl, Adrian Friday, A. J. Brush, and Yoshito Tobe (Eds.). Springer-Verlag, Berlin, Heidelberg, 95-114.

DOI=http://dx.doi.org/10.1007/978-3-642-01516-8_8

10. Eric Horvitz. 1999. Principles of mixed-initiative user interfaces. In Proceedings of the SIGCHI conference on Human Factors in Computing Systems (CHI '99). ACM, New York, NY, USA, 159-166. DOI=http://dx.doi.org/10.1145/302979.303030

11. Chuan-Che (Jeff) Huang, Rayoung Yang, and Mark W. Newman. 2015. The potential and challenges of inferring thermal comfort at home using commodity sensors. In Proceedings of the 2015 ACM International Joint Conference on Pervasive and Ubiquitous Computing (UbiComp '15). ACM, New York, NY, USA, 1089-1100.

DOI=http://dx.doi.org/10.1145/2750858.2805831.

12. Stephen S. Intille. 2002. Designing a Home of the Future. IEEE Pervasive Computing 1, 2 (April 2002), 76-82.

DOI=http://dx.doi.org/10.1109/MPRV.2002.1012340

13. Xiaofan Jiang, Stephen Dawson-Haggerty, Prabal Dutta, and David Culler. 2009. Design and implementation of a high-fidelity AC metering network. In Proceedings of the 2009 International Conference on Information Processing in Sensor Networks (IPSN '09). IEEE Computer Society, Washington, DC, USA, 253-264.

14. Antti Jylhä, Petteri Nurmi, Miika Sirén, Samuli Hemminki, and Giulio Jacucci. 2013. MatkaHupi: a persuasive mobile application for sustainable mobility. In Proceedings of the 2013 ACM conference on Pervasive and ubiquitous computing adjunct publication (UbiComp '13 Adjunct). ACM, New York, NY, USA, 227-230. DOI=http://dx.doi.org/10.1145/2494091.2494164

15. Richard Katzev, Laura Cooper, and Pat Fisher. 1981. Effect of feedback and social reinforcement on residential electricity consumption. Journal of Environmental Systems. 10, 3: 215-227.

16. Christian Koehler, Brian D. Ziebart, Jennifer Mankoff, and Anind K. Dey. 2013. TherML: occupancy prediction for thermostat control. In Proceedings of the 2013 ACM International Joint Conference on Pervasive and Ubiquitous Computing (UbiComp '13). ACM, New York, NY, USA, 103-112. DOI=http://dx.doi.org/10.1145/2493432.2493441

17. Lenneke Kuijer and Annelise De Jong. 2012. Identifying design opportunities for reduced household resource consumption: exploring practices of thermal comfort. Journal of Design Research 10, 1-2: 67-85. DOI: http://dx.doi.org/10.1504/JDR.2012.046140

18. Amanda Lazar, Christian Koehler, Joshua Tanenbaum, and David H. Nguyen. 2015. Why we use and abandon smart devices. In Proceedings of the 2015 ACM International Joint Conference on Pervasive and 
Ubiquitous Computing (UbiComp '15). ACM, New York, NY, USA, 635-646. DOI=http://dx.doi.org/10.1145/2750858.2804288

19. Jiakang Lu, Tamim Sookoor, Vijay Srinivasan, Ge Gao, Brian Holben, John Stankovic, Eric Field, and Kamin Whitehouse. 2010. The smart thermostat: using occupancy sensors to save energy in homes. In Proceedings of the 8th ACM Conference on Embedded Networked Sensor Systems (SenSys '10). ACM, New York, NY, USA, 211-224. DOI=http://doi.acm.org/10.1145/1869983.1870005

20. Sarah Mennicken and Elaine M. Huang. 2012. Hacking the natural habitat: an in-the-wild study of smart homes, their development, and the people who live in them. In Proceedings of the 10th international conference on Pervasive Computing (Pervasive'12), Judy Kay, Paul Lukowicz, Hideyuki Tokuda, Patrick Olivier, and Antonio Krüger (Eds.). Springer-Verlag, Berlin, Heidelberg, 143-160.

DOI=http://dx.doi.org/10.1007/978-3-642-31205-2_10

21. Michael C. Mozer. 1998. The neural network house: An environment hat adapts to its inhabitants. In Proceedings of the AAAI Spring Symp. Intelligent Environments, Vol. 58. 1998.

22. J. Fergus Nicol and Michael A. Humphreys. 2002. Adaptive thermal comfort and sustainable thermal standards for buildings. Energy and buildings 34, 6: 563-572. DOI=http://dx.doi.org/10.1016/S03787788(02)00006-3

23. James Pierce, Chloe Fan, Derek Lomas, Gabriela Marcu, and Eric Paulos. 2010. Some consideration on the (in)effectiveness of residential energy feedback systems. In Proceedings of the 8th ACM Conference on Designing Interactive Systems (DIS '10). ACM, New York, NY, USA, 244-247. DOI=http://dx.doi.org/10.1145/1858171.1858215

24. Devika Pisharoty, Rayoung Yang, Mark W. Newman, and Kamin Whitehouse. 2015. ThermoCoach: Reducing Home Energy Consumption with Personalized Thermostat Recommendations. In Proceedings of the 2nd ACM International Conference on Embedded Systems for Energy-Efficient Built Environments (BuildSys '15). ACM, New York, NY, USA, 201-210. DOI=http://dx.doi.org/10.1145/2821650.2821671

25. Juhi Ranjan, Erin Griffiths, and Kamin Whitehouse. 2014. Discerning electrical and water usage by individuals in homes. In Proceedings of the 1st ACM Conference on Embedded Systems for Energy-Efficient Buildings (BuildSys '14). ACM, New York, NY, USA, 20-29.

DOI=http://dx.doi.org/10.1145/2674061.2674066
26. James Scott, A.J. Bernheim Brush, John Krumm, Brian Meyers, Michael Hazas, Stephen Hodges, and Nicolas Villar. 2011. PreHeat: controlling home heating using occupancy prediction. In Proceedings of the 13th international conference on Ubiquitous computing (UbiComp '11). ACM, New York, NY, USA, 281-290. DOI=http://dx.doi.org/10.1145/2030112.2030151

27. Patrick C. Shih, Kyungsik Han, Erika Shehan Poole, Mary Beth Rosson, and John M. Carroll. 2015. Use and Adoption Challenges of Wearable Activity Trackers. iConference 2015 Proceedings.

28. Will Simm, Maria Angela Ferrario, Adrian Friday, Peter Newman, Stephen Forshaw, Mike Hazas, and Alan Dix. 2015. Tiree Energy Pulse: Exploring Renewable Energy Forecasts on the Edge of the Grid. In Proceedings of the 33rd Annual ACM Conference on Human Factors in Computing Systems (CHI '15). ACM, New York, NY, USA, 1965-1974. DOI: http://dx.doi.org/10.1145/2702123.2702285

29. Yolande Strengers. 2011. Designing eco-feedback systems for everyday life. In Proceedings of the SIGCHI Conference on Human Factors in Computing Systems (CHI '11). ACM, New York, NY, USA, 2135 2144.

DOI=http://dx.doi.org/10.1145/1978942.1979252

30. Yolande Strengers. 2014. Smart energy in everyday life: are you designing for resource man?. interactions 21, 4 (July 2014), 24-31. DOI=10.1145/2621931 http://doi.acm.org/10.1145/2621931

31. Rayoung Yang and Mark W. Newman. 2013. Learning from a learning thermostat: lessons for intelligent systems for the home. In Proceedings of the 2013 ACM international joint conference on Pervasive and ubiquitous computing (UbiComp '13). ACM, New York, NY, USA, 93-102. DOI=http://dx.doi.org/10.1145/2493432.2493489

32. Rayoung Yang, Mark W. Newman, and Jodi Forlizzi. 2014. Making sustainability sustainable: challenges in the design of eco-interaction technologies. In Proceedings of the SIGCHI Conference on Human Factors in Computing Systems (CHI '14). ACM, New York, NY, USA, 823-832. DOI=http://dx.doi.org/10.1145/2556288.2557380

33. Nest. The Learning Thermostat. 2016. Retrieved February 16, 2016 from https://nest.com

34. Pet Industry Market Size \& Ownership Statistics 2016. Retrieved February 16, 2016 from http://www.americanpetproducts.org/press_industrytre nds.asp 